OPEN ACCESS

Edited by:

Phuc Huu Phan

Vietnam National Hospital of

Pediatrics, Vietnam

Reviewed by:

Claudia Schweiger,

Clinical Hospital of Porto Alegre, Brazil

Narayanan Parameswaran,

Jawaharlal Institute of Postgraduate

Medical Education and Research

(JIPMER), India

*Correspondence:

Suresh Kumar Angurana

sureshangurana@gmail.com

Specialty section:

This article was submitted to

Pediatric Critical Care

a section of the journal

Frontiers in Pediatrics

Received: 23 May 2021

Accepted: 26 July 2021

Published: 16 September 2021

Citation:

Kumar V, Angurana SK, Baranwal AK and Nallasamy K (2021) Nasotracheal

vs. Orotracheal Intubation and

Post-extubation Airway Obstruction in Critically III Children: An Open-Labe

Randomized Controlled Trial.

Front. Pediatr. 9:713516

doi: 10.3389/fped.2021.713516

\section{Nasotracheal vs. Orotracheal Intubation and Post-extubation Airway Obstruction in Critically III Children: An Open-Label Randomized Controlled Trial}

\author{
Vijay Kumar, Suresh Kumar Angurana*, Arun Kumar Baranwal and Karthi Nallasamy \\ Division of Pediatric Critical Care, Department of Pediatrics, Advanced Pediatric Centre, Postgraduate Institute of Medical \\ Education and Research, Chandigarh, India
}

Background: The data on long-term nasotracheal intubation among mechanically ventilated critically ill children is limited. The purpose of this study was to compare the rate of post-extubation airway obstruction (PEAO) with nasotracheal and orotracheal intubation.

Methods: This open-label randomized controlled trial was conducted in PICU of a tertiary care and teaching hospital in North India from January-December 2020 involving intubated children aged 3 months-12 years. After written informed consent, children were randomized into nasotracheal and orotracheal intubation groups. Post-extubation, modified Westley's croup score (mWCS) was used at 10-timepoints (0-min, 30 min, 1, 2, $3,6,12,24,36$, and 48-h after extubation) to monitor for PEAO. The primary outcome was the rate of PEAO; and secondary outcomes were time taken for intubation, number of intubation attempts, complications during intubation, unplanned extubation, repeated intubations, tube malposition/displacement, endotracheal tube blockade, ventilator associated pneumonia, skin trauma, extubation failure/re-intubation, duration of PICU stay, and mortality.

Results: Seventy children were randomized into nasotracheal $(n=30)$ and orotracheal $(n=40)$ groups. Both the groups were similar in baseline characteristics. The rate of PEAO was similar between nasotracheal and orotracheal groups (10 vs. 20\%, $p=0.14$ ). The maximum mWCS and mWCS at 10-timepoints were similar in two groups. The time taken for intubation was significantly longer ( 85 vs. 48 s, $p<0.001$ ) in nasotracheal group, whereas other secondary outcomes were similar in two groups.

Conclusion: The rate of PEAO was not different between nasotracheal and orotracheal groups.

Clinical Trial Registration: http://ctri.nic.in, Identifier: CTRI/2020/01/022988.

Keywords: post-extubation stridor, extubation, airway edema, reintubation, post-extubation airway obstruction, nasotracheal intubation 


\section{INTRODUCTION}

Endotracheal intubation is commonly performed intervention in critically ill children to provide mechanical ventilation in emergency rooms (ERs) and Pediatric intensive care units (PICUs). Orotracheal and nasotracheal intubation are two modes with their own advantages and disadvantages (1-3). Orotracheal intubation is generally preferred and commonly used as it is easier, quicker especially during emergent intubations, and less painful $(1,2)$. Nasotracheal intubation is commonly used in operating rooms especially during dental, oropharyngeal, and maxillofacial surgeries as it is easier to ventilate the patient and administer anesthetic gases without limiting access to oral cavity and oropharynx $(1,2)$. Nasotracheal intubation has several advantages as it is easier to secure; moves less, if secured properly; lesser risk of trauma to lips, tongue and larynx; lesser chances of unplanned extubation; more patient comfort; and possibly lower rate of post-extubation airway obstruction (PEAO) $(1,2)$. However, nasotracheal intubation can cause injury (to nose, turbinate, and nasopharynx), bleeding, and increases the risk of sinusitis $(1,2,4-10)$. As nasotracheal intubation is technically challenging and associated with more complications, it is recommended that it should be performed by skilled healthcare providers $(1,2)$. Due to these reasons, nasotracheal intubation is less commonly practiced (2-5.6\% of all endotracheal intubations) among adults and children undergoing mechanical ventilation in ICUs $(2,5,11-15)$.

Few studies involving critically ill children on mechanical ventilation documented lower rate of unplanned extubation in nasotracheal group than in orotracheal intubation group (11, 16-18). However, the literature on the long-term nasotracheal intubation among mechanically ventilated critically ill children and its impact on PEAO is not available, despite the theoretical benefits of nasotracheal intubation. Therefore, we conducted this study to compare the nasotracheal and orotracheal routes of endotracheal intubation among mechanically ventilated critically ill children and compared the rate of PEAO between the two groups.

\section{METHODOLOGY}

This open-label randomized controlled trial was conducted in a 15-bedded PICU of a tertiary care teaching hospital in North India over a period of 1 year (January 2020 to December 2020) including children aged 3 months -12 years with endotracheal intubation and invasive mechanical ventilation. The children with tracheostomy, raised intracranial pressure, severe acute respiratory distress syndrome (ARDS), refractory septic shock, remained intubated in ER for $>24 \mathrm{~h}$, referred intubated to ER from peripheral hospitals, anticipated intubation $<24 \mathrm{~h}$, cases requiring re-intubation after one episode of mechanical ventilation, known bleeding disorder, recent nasal surgery or trauma or burns, previous history of epistaxis, chronic lung disease, congenital heart disease, and with nasal and other facial malformation were excluded. The study protocol was approved by the Institute Ethics Committee (PGI/IEC/2019/002796, dated 28-12-2019) and registered with the Clinical Trials
Registry-India (CTRI/2020/01/022988). The written informed consent was obtained from the parents/legal guardian before enrolment.

\section{Randomization}

Patients were enrolled on the day of admission to PICU or whenever intubation was performed in PICU. The eligible children were randomized into 2 groups (nasotracheal orotracheal intubation groups) by using computer generated randomization table. The slips mentioning the group were placed in serially numbered, sealed, and opaque envelops which were opened at the time of randomization by the primary investigator (VK).

\section{Intubation Procedure}

In our unit, we routinely perform orotracheal intubation. Children randomized to nasotracheal group were re-intubated through the nasal route. The primary investigator and senior residents working in the unit were trained in performing orotracheal and nasotracheal intubation. The standard protocol was followed to perform orotracheal and nasotracheal intubation. Adequate sedation, analgesia, and neuromuscular blockade (if needed) were used. Children were pre-oxygenated with bag and mask ventilation. The size (in $\mathrm{mm}$ ) of endotracheal tube (ETT) was calculated as per the standard formulae for uncuffed (Age in years $/ 4+4$ ) and cuffed tube (Age in years $/ 4+3.5$ ). The length (in $\mathrm{cm}$ ) of insertion of ETT was calculated as ETT size $\mathrm{x} 3$ or Age in years $/ 2+12$ for orotracheal intubation and Age in years/2 +15 for nasotracheal intubation $(1,19)$. We used micro-cuffed endotracheal tubes in all cases.

For nasotracheal intubation, the lidocaine jelly (as local anesthetic and lubricant) was applied to the nasal cavity and ETT prior to intubation. The ETT was then passed through nares into nasopharynx under direct laryngoscopy. Once it reached nasopharynx, it was guided into the glottic opening by using Magill's forceps $(1,2)$. During the procedure, oxygen saturation and heart rate was monitored continuously and time taken for intubation (in seconds) was recorded. The appropriate position of ETT was confirmed by clinical examination (auscultation over stomach and bilateral axilla) and later on by the chest radiograph, as per routine in the unit. The ETT was secured by the using dynaplast. For orotracheal intubation, one strip of dynaplast was pasted to the upper lip and another E-shaped strip was used to secure tube to upper and lower lip. For nasotracheal intubation, one strip of dynaplast was pasted to upper lip and another-Y-shaped strip was used to secure tube to upper lip. Any repositioning of the ETT after intubation was also documented.

\section{General Care}

All children were managed and monitored as per unit's existing protocol for management of critically ill children for intubation, mechanical ventilation, sedation and analgesia, hemodynamic monitoring and treatment, nutrition, nursing support, weaning, extubation, and post-extubation care. Routine nursing care was provided in form of strict aseptic precautions, minimal handling, proper fixation of tube, clustering of interventions, and frequent position changes (if not contraindicated). The suction 
of endotracheal tube was done every 4-6-h or whenever needed. Enteral feeding was started as soon as possible, preferably within $24 \mathrm{~h}$ of admission to the PICU. Among children intubated for $>48 \mathrm{~h}$, six dosage of dexamethasone $(0.5 \mathrm{mg} / \mathrm{kg} / \mathrm{dose})$ were used peri-extubation, with first dose given $24 \mathrm{~h}$ prior to extubation $(20,21)$. Feeding was withheld for $6 \mathrm{~h}$ prior to extubation and 4-6 h after extubation.

\section{Post-extubation Monitoring}

We used modified Westley's croup score (mWCS) to monitor for PEAO at 10-timepoints (0-, 30-min, 1, 2, 3, 6, 12, 24, 36, and 48-h after extubation) (Supplementary Table 1) (20, $22,23)$. A $m W C S \geq 4$ suggested administration of adrenaline nebulization ( $1 \mathrm{mg} / \mathrm{ml} ; 2.5 \mathrm{ml}$ in $2.5 \mathrm{ml}$ saline every $20 \mathrm{~min}$ until improvement). The re-intubation (by oronasal route) was performed if there was no response after adrenaline nebulization as evident by audible stridor, marked decreased air entry, severe chest indrawing and/or respiratory acidosis $(\mathrm{pH}<7.35$ and $\mathrm{PaCO}_{2}>45 \mathrm{mmHg}$ ), $\mathrm{SpO}_{2}<90 \%$ at $\mathrm{FiO}_{2}>40 \%$, bradycardia, or other clinical sign of impending respiratory failure, or mWCS of 7 (extubation failure) (20).

\section{Data Collection}

Baseline data (age, sex, diagnosis), admission Glasgow Coma Scale (GCS), pediatric risk of mortality III (PRISM III) score, maximum vasoactive inotropic score (VIS), and sequential organ failure assessment (SOFA) score on day 1,2 , and 7 were noted. Time taken for intubation, number of intubation attempts, complications during intubation (hypoxemia, bradycardia, hypotension or cardiac arrest), unplanned or accidental extubation, repeated intubations, tube malposition or displacement, ETT blockade, skin trauma related to ETT, epistaxis, sinusitis, healthcare associated infections (HCAIs), ventilator associated pneumonia (VAP), post-extubation atelectasis, extubation failure/reintubation, duration of intubation, duration of PICU stay, and final outcome (survival or death) were recorded.

\section{Definitions}

The clinically significant $P E A O$ was defined as $\mathrm{mWCS} \geq 4$. Time taken for intubation was defined as period from stopping the bag and mask ventilation to starting positive pressure ventilation after insertion of ETT. Intubation failure was considered if there was bradycardia (heart rate $<60 / \mathrm{min})$ and/or desaturation $\left(\mathrm{SPO}_{2}\right.$ $<90 \%$ ) or both during the intubation attempt. Numbers of intubation attempts defined as number of times procedure was aborted and requiring re-oxygenation and another attempt to intubate. The standard definitions were used for Sepsis and severe sepsis (24), and VAP (25). The skin trauma related to ETT was classified as per the standardized classification of decubitus lesions by the US National Pressure Ulcer Advisory Panel (NPUAP) (26).

\section{Outcomes}

The primary outcome was the rate of PEAO among children in nasotracheal and orotracheal groups. Secondary outcomes were time taken for intubation, number of intubation attempts, complications during intubation, unplanned extubation, repeated intubations, tube malposition/displacement, ETT blockade, VAP, skin trauma related to ETT (injury to skin, nostrils, nasal septum, lip, or tongue), extubation failure/reintubation, duration of PICU stay, and survival or death.

\section{DATA ENTRY AND STATISTICAL ANALYSIS}

The sample size was calculated based on the incidence of PEAO (32.8-34\%) documented in previous studies from our PICU $(21,23)$. As a superiority trial, with the incidence of PEAO in nasotracheal group as $15 \%$ and $\beta$-error of 0.2 , the required sample size was 90 cases in each group $(n=180)$.

However, in view of COVID-19 situation, the number of admissions to the PICU and those underwent mechanical ventilation were reduced (585 admissions during study period as compared to 900-100 admissions per year in normal times), leading to slower recruitment. The study was stopped after the end of the study period (with enrolment of 70 cases) as it was a dissertation project of a Pediatric Critical Care Fellow (VK), and the dissertations are time bound in our institute. The Dean of the institute approved to go ahead with the sample size of 70 (letter no. 12396/1TRG/PG-2019/15029, dated 16/12/2020).

Data entry and statistical analysis were performed using Microsoft Excel 2013 (Microsoft, Redmond, WA) and SPSS software version 21(IBM Corp. 2012. IBM SPSS Statistics for Windows, Version 21.0. Armonk, NY: IBM Corp). Descriptive

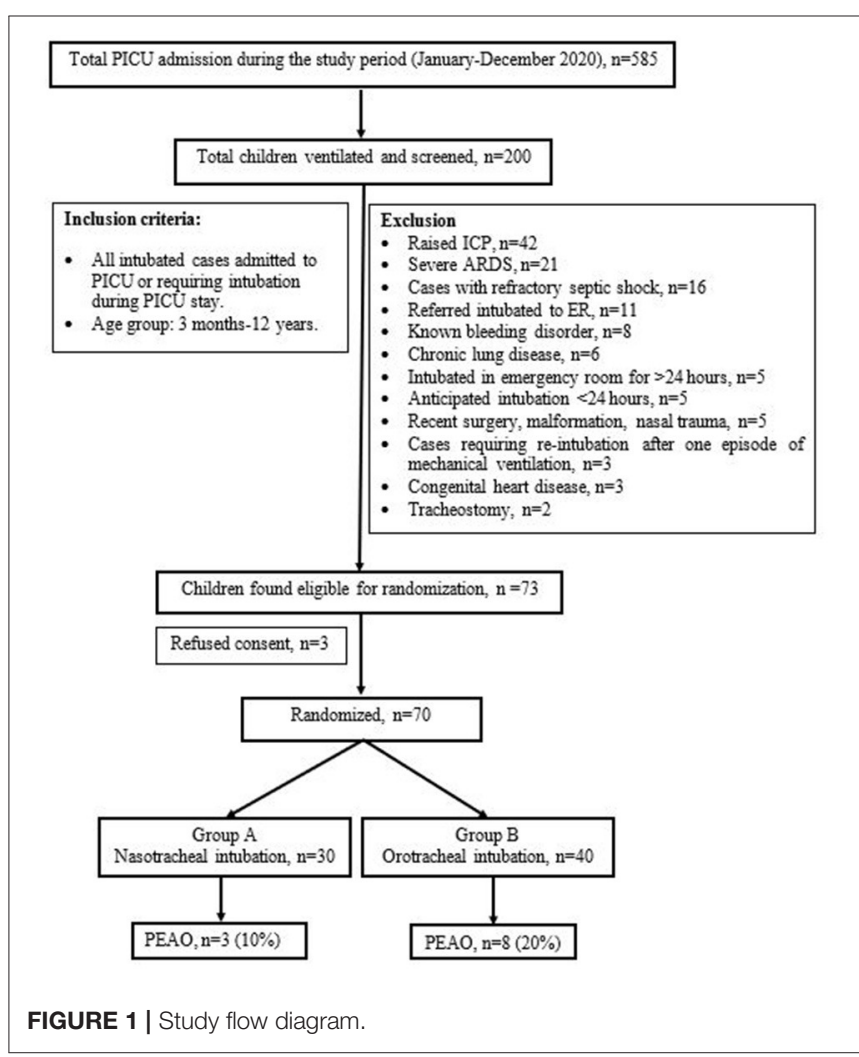


TABLE 1 | Baseline characteristics and severity scores among children in nasotracheal and orotracheal intubation groups.

\begin{tabular}{|c|c|c|c|c|}
\hline Patient characteristics & $\begin{array}{c}\text { Total } \\
(n=70)\end{array}$ & $\begin{array}{l}\text { Nasotracheal } \\
\qquad(n=30)\end{array}$ & $\begin{array}{l}\text { Orotracheal } \\
\qquad(n=40)\end{array}$ & $P$ \\
\hline Male, $n(\%)$ & $41(58.6)$ & $19(63)$ & $22(55)$ & 0.48 \\
\hline Age (month); median (IQR) & $36(12-96)$ & $42(21-133)$ & $30(11-85)$ & 0.73 \\
\hline \multicolumn{5}{|l|}{ Diagnosis } \\
\hline Snake envenomation, $n$ (\%) & $13(18.6)$ & $9(30)$ & $4(10)$ & 0.12 \\
\hline LGBS, $n(\%)$ & $8(11.4)$ & $3(10)$ & $5(12.5)$ & \\
\hline CNS infections, $n(\%)$ & $7(10)$ & $3(10)$ & $4(10)$ & \\
\hline Scrub typhus, $n(\%)$ & $7(10)$ & $2(6.7)$ & $5(12.5)$ & \\
\hline Poisoning, $n(\%)$ & $7(10)$ & $3(10)$ & $4(10)$ & \\
\hline ARDS, $n(\%)$ & $5(7.1)$ & $1(3.3)$ & $4(10)$ & \\
\hline Tetanus, $n(\%)$ & $1(1.4)$ & $1(3.3)$ & 0 & \\
\hline Site of intubation prior to enrolment & & & & 0.84 \\
\hline $\mathrm{ER}, n(\%)$ & $60(85.7)$ & $26(86.7)$ & $34(85)$ & \\
\hline $\mathrm{PICU}, n(\%)$ & $10(14.3)$ & $4(13.3)$ & $6(15)$ & \\
\hline GCS at admission, median (IQR) & $8(6-10)$ & $7(5-10)$ & $9(8-12)$ & 0.02 \\
\hline PRISM III Score, median (IQR) & $13(9-20)$ & $12(9-15)$ & $16(11-20)$ & 0.09 \\
\hline Maximum VIS score, median (IQR) & $50(20-62)$ & $43(13-53)$ & $50(21-65)$ & 0.73 \\
\hline \multicolumn{5}{|l|}{ SOFA score, median (IQR) } \\
\hline Day 1 & $6(4-9)$ & $5(2-6)$ & $8(4-9)$ & 0.07 \\
\hline Day 2 & $3(2-8.5)$ & $3(2-4.2)$ & $4(2-9)$ & 0.07 \\
\hline Day 7 & $1(0-2)$ & $1(0-2)$ & $1(0-5)$ & 0.42 \\
\hline
\end{tabular}

statistics [mean (SD), median (IQR), range, number, and percentages] were used for baseline variables. Dichotomous outcomes were compared by chi-square test or Fisher's exact-test, as applicable. Continuous variables were compared by Student $t$-test or Mann-Whitney $U$-test. The repeated measure analysis of variance (RM-ANOVA) was used to compare mWCS between 2 groups over 10-timepoints. All tests used were two-tailed and $p$-value $<0.05$ was taken as significant.

\section{RESULTS}

During the study period, there were 585 admissions to the PICU, 200 (34.2\%) were ventilated, and 70 were randomized to nasotracheal $(n=30)$ and orotracheal $(n=40)$ groups (Figure 1).

\section{Baseline Characteristics}

There were $58.7 \%(n=41)$ males with median (IQR) age of 36 (12-96) months. The most common diagnosis included snake envenomation (18.6\%), metabolic disorder (12.9\%), sepsis (11.4\%), Landry-Guillain-Barre syndrome (11.4\%), central nervous system infections (10\%), Scrub typhus (10\%), poisoning (10\%), and ARDS (7.1\%). The initial orotracheal intubation was performed in ER among $85.7 \%$ cases and in PICU among $14.3 \%$ cases. Later, the cases in nasotracheal group were extubated and reintubated through the nasal route. The median GCS at admission was 8 (6-10), PRISM-III score was 13 (9-20), and maximum VIS score was 50 (20-62). The SOFA score on day 1,2 , and 7 were 6 (4-9), 3 (2-8.5), and 1 (0-2), respectively. Both the groups were comparable as far as baseline variables are concerned. However, children in nasotracheal group had lower GCS at admission $(p=0.02)$ (Table 1).

\section{Primary Outcome}

The overall rate of PEAO was $15 \%(n=11)$. The rate of PEAO in nasotracheal and orotracheal groups was $10 \%$ $(n=3)$ and $20 \%(n=8)$, respectively $(p=0.14)$. The maximum mWCS [mean (SE)] was $1.81(0.25)$ and it was comparable in nasotracheal and orotracheal groups [1.62 (0.38) vs. 1.98 (0.33), respectively, $p=0.47$ ] (Table 2). The serial mWCS in the first $48 \mathrm{~h}$ following extubation was also similar in two groups (Table 2; Figure 2). The RM-ANOVA showed no significant difference in mWCS between 2 groups over 10-timepoints $(p=0.53$, Wilks Lambda Test).

\section{Secondary Outcomes}

The overall time taken [median (IQR)] for intubation was 60 (47-85) s and it was significantly higher in nasotracheal group as compared to orotracheal group [ 85 (75-90) s vs. 48 (45-60) s, respectively, $p<0.001]$. Other outcomes like children requiring 
TABLE 2 | Primary outcomes in nasotracheal and orotracheal intubation groups.

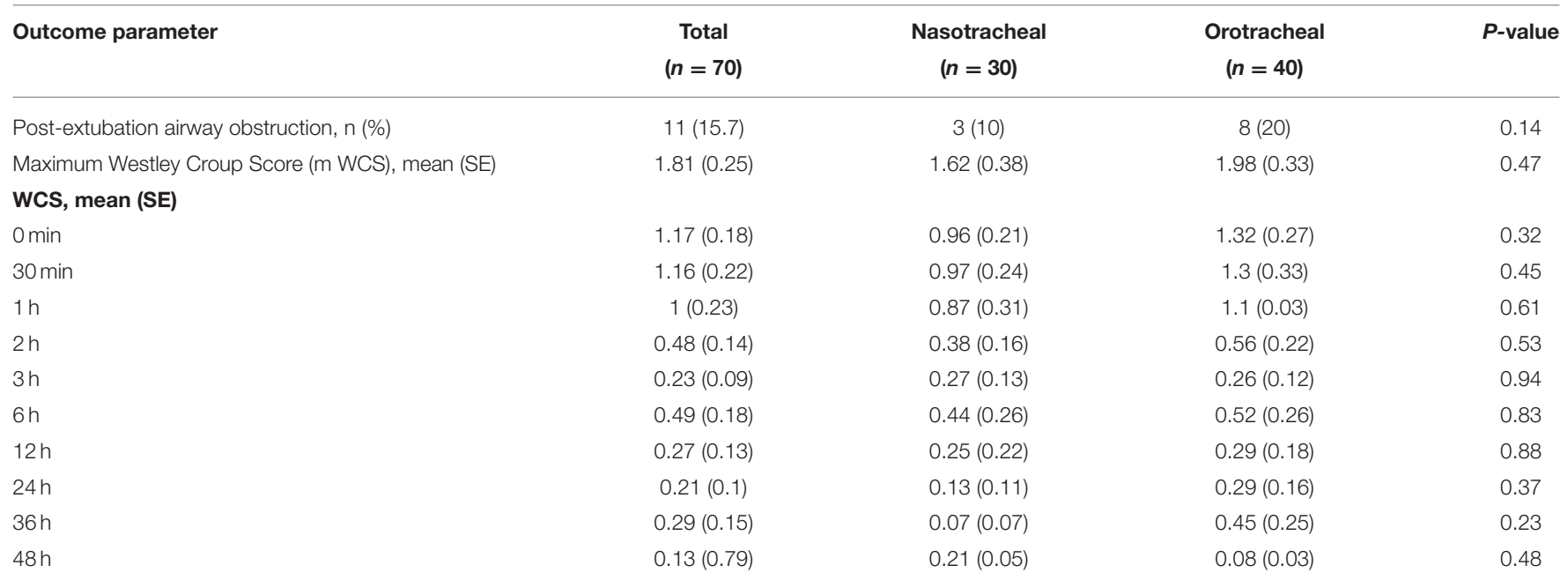

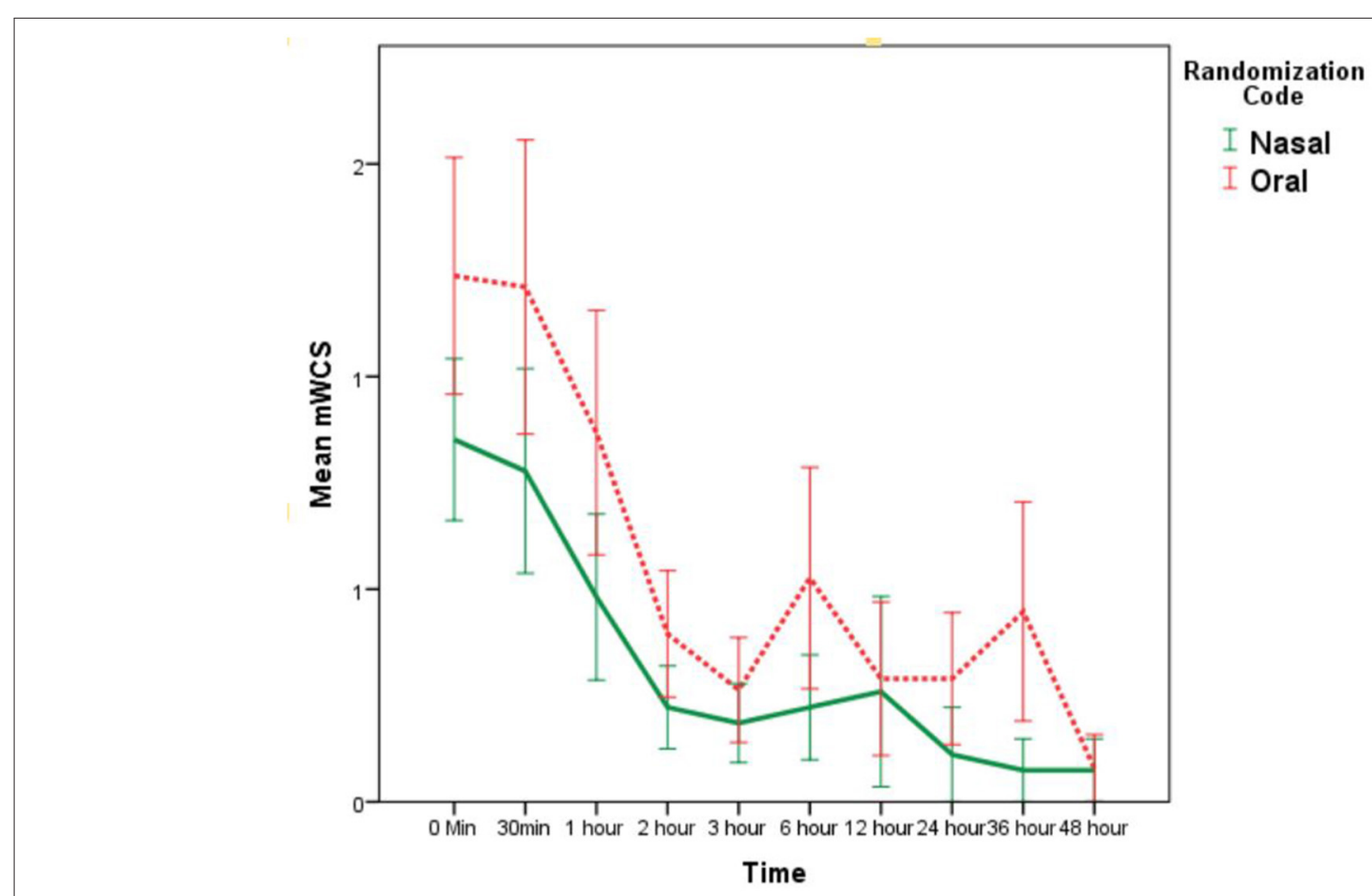

FIGURE 2 | mWCS [mean (SE)] in nasotracheal and orotracheal intubation groups at different time points after extubation.

$>1$ intubation attempt (10 vs. $2.5 \%)$, complications during intubation (3.3 vs. $2.5 \%$ ), unplanned extubation (10 vs. $15 \%$ ), repeated intubation (10 vs. $15 \%)$, tube malposition/displacement (6.7 vs. 5\%), ETT blockade (0 vs. $7.5 \%)$, skin trauma (10 vs. $5 \%$ ), VAP (6.7 vs. $5 \%)$, duration of intubation (6.5 vs. 7 days), adrenaline nebulization (10 vs. $20 \%)$, post-extubation atelctasis ( $10 \mathrm{vs.} 0 \%)$, type of post-extubation respiratory support, extubation failure/reintubation (6.7 vs. 8.5), duration of PICU stay ( 7.5 vs. 9 days), and mortality (6.7 vs. $12.5 \%$ ) were similar in two groups (Table 3). The time of onset of PEAO in two groups was also similar $(p=0.22$, Log Rank test).

\section{DISCUSSION}

In this open-label RCT, we noted that in critically ill children undergoing mechanical ventilation, the rate of PEAO (10 vs. $20 \%)$ and maximum mWCS (1.62 vs. 1.98$)$ were similar in nasotracheal and orotracheal intubation groups. The serial mWCS was also similar in two groups during the first $48 \mathrm{~h}$ after extubation. The rate of PEAO (15\%) in the index study was within the range of the documented rates of PEAO among critically ill children (18-40\%) (20, 23, 27-29). However, the rate of PEAO in index study was lower than the reported rates of 
TABLE 3 | Secondary and final outcomes in nasotracheal and orotracheal intubation groups.

\begin{tabular}{|c|c|c|c|c|}
\hline Outcome parameter & $\begin{array}{c}\text { Total } \\
(n=70)\end{array}$ & $\begin{array}{l}\text { Nasotracheal } \\
\qquad(n=30)\end{array}$ & $\begin{array}{l}\text { Orotracheal } \\
\qquad(n=40)\end{array}$ & $P$-value \\
\hline $\begin{array}{l}\text { Time taken for intubation (seconds); } \\
\text { median (IQR) }\end{array}$ & $60(47-85)$ & $85(75-90)$ & $48(45-60)$ & $<0.001$ \\
\hline Intubation attempts >1, $n$ (\%) & $4(5.7)$ & $3(10)$ & $1(2.5)$ & 0.18 \\
\hline Complication during Intubation & $2(2.8)$ & $1(3.3)$ & $1(2.5)$ & 0.84 \\
\hline \multicolumn{5}{|l|}{ Hypoxia/Bradycardia, $n$ (\%) } \\
\hline Unplanned extubation, $n(\%)$ & $9(12)$ & $3(10)$ & $6(15)$ & 0.54 \\
\hline Repeated intubation, $n$ (\%) & $9(12)$ & $3(10)$ & $6(15)$ & 0.28 \\
\hline Tube malposition/displacement, $n$ (\%) & $4(5.7)$ & $2(6.7)$ & $2(5)$ & 0.77 \\
\hline Endotracheal tube blockade, $n(\%)$ & $3(4.2)$ & $0(0)$ & $3(7.5)$ & 0.13 \\
\hline Skin trauma related to ET, $n(\%)$ & $5(7.1)$ & $3(10)$ & $2(5)$ & 0.42 \\
\hline VAP, $n(\%)$ & $3(4.3)$ & $2(6.7)$ & $1(5)$ & 0.14 \\
\hline Duration of intubation, median (IQR) & $7(3-13)$ & $6.5(3-13)$ & $7(3-13)$ & 0.81 \\
\hline Adrenaline nebulization, $n(\%)$ & $11(15.7)$ & $3(10)$ & $8(20)$ & 0.14 \\
\hline Post-extubation atelectasis, $n$ (\%) & $3(4.3)$ & $3(10)$ & $0(0)$ & 0.08 \\
\hline \multicolumn{5}{|l|}{ Post-extubation respiratory support } \\
\hline Nasal prongs, $n(\%)$ & $14(20)$ & $6(20)$ & $8(20)$ & 0.62 \\
\hline Nasal CPAP, $n(\%)$ & $29(41.4)$ & $15(50)$ & $14(35)$ & \\
\hline BiPAP, $n(\%)$ & $13(18.7)$ & $5(16.7)$ & $8(20)$ & \\
\hline High flow nasal cannula, $n(\%)$ & $2(2.8)$ & $1(3.0)$ & $1(1.4)$ & \\
\hline Extubation failure $n(\%)$ & $5(7.1)$ & $2(6.7)$ & $3(7.5)$ & 1 \\
\hline Duration of PICU stay, median (IQR) & $8(5-13)$ & $7.5(4.7-14)$ & $9(5-13)$ & 0.77 \\
\hline Death, $n(\%)$ & $7(10)$ & $2(6.7)$ & $5(12.5)$ & 0.69 \\
\hline
\end{tabular}

PEAO in the recent studies from our unit $(32.8-34 \%)(21,23)$. The lower rate of PEAO could be due to the fact that we used micro-cuffed endotracheal tubes (high-volume-low-pressure) in all cases as these were routinely available from the hospital supply during the study period. The use of micro-cuffed ETT may had led to lesser movement of ETT, lesser chances of unplanned extubation or ETT change, lower risk of laryngeal edema, and hence lower rates of $\operatorname{PEAO}(15,30,31)$. None of the Pediatric studies looked into the impact of nasotracheal intubation on the rate of PEAO, time taken for intubation, unplanned extubation, extubation failure, and other important clinical outcomes (duration of PICU stay and mortality).

We noted that the nasotracheal intubation took more time than the orotracheal intubation, as it is technically more complex. However, other outcomes like children requiring $>1$ intubation attempt, complications during intubation, unplanned extubation, repeated intubation, tube malposition/displacement, ETT blockade, skin trauma, VAP, duration of intubation, adrenaline nebulization, post-extubation atelctasis, postextubation respiratory support, extubation failure, duration of PICU stay, and mortality were similar in two groups. Previous studies also demonstrated that time taken for nasotracheal intubation was significantly longer than orotracheal intubation among critically ill adults and children (32-34). Also, nasotracheal intubation when compared to orotracheal intubation was associated with more changes in heart rate and blood pressure in early post-intubation period (33); need of more number of additional providers, more intubation attempts, and more traumatic intubations (34).
The literature on the outcome of long-term nasotracheal intubation in children on mechanical ventilation is limited. Spence and Barr (35) conducted a systematic review involving 2 randomized trials that compared nasal vs. oral intubation in neonates requiring mechanical ventilation and demonstrated that there were no differences between the orotracheal and nasotracheal route of intubation. One study noted higher rate of intubation failure using the nasal route; and one noted higher rates of post-extubation atelectasis in nasally intubated neonates weighing $<1,500 \mathrm{~g}$. The rates of ETT malposition, accidental extubation, tube blockage, re-intubation after extubation, septicemia, clinical infection, and local trauma were similar between two groups. Recently, Christian et al. (11) published a retrospective cohort study (January 2015 to December 2016) involving 121 PICUs in the United States and noted that $53 \%(n=64)$ of PICUs had zero nasotracheal intubations during the study period. Out of 12,088 endotracheal intubations, only 5.6\% $(n=680)$ were nasotracheal. Among nasotracheal group, the rate of unplanned extubation was significantly less as compared to orotracheal group (0.9 vs. $2.9 \%$, $p<0.001)$. However. The rates of sinusitis and VAP were similar in two groups. Among children in nasotracheal group, majority were $<2$ years $(88.1 \%$ ), and $82.2 \%$ were classified as cardiac cases. Among young cardiac cases, the rate of unplanned extubation was significantly lower in nasotracheal group as compared to orotracheal group ( 0 vs. $2.1 \%, p<0.001$ ).

Unplanned extubation is one of the serious adverse events noted in cases with endotracheal intubation and associated with increased mortality, duration of mechanical ventilation, 
and ICU stay $(16,18,36,37)$. As ETT is well-secured with nasotracheal intubation, the chances of unplanned extubation are lesser, which has been demonstrated among adults and children (17, 38, 39). However, Piva et al. (40) demonstrated that among children in PICU, the rate of unplanned extubation was similar in orotracheal and nasotracheal group (3.1 vs. $1.6 \%$, respectively, $p=0.06$ ). Nasotracheal ETT can lead to blockage of drainage of paranasal sinuses, local trauma, edema, and local infection of nasal mucosa which can leads to sinusitis. The nasotracheal intubation has been identified as an important risk factor for sinusitis among adults and children (5-10). Moreover, the sinusitis can evolve into sepsis, bacteremia, and VAP (41). The rate of unplanned extubation was similar in two groups in the index study and none had sinusitis.

\section{Strength and Limitations}

This is the first RCT that compared the nasotracheal vs. orotracheal route of endotracheal intubation in critically ill children receiving invasive mechanical ventilation. All enrolled cases were analyzed for the final outcome. We uniformly used micro-cuffed ETT in all cases. The limitations of this study include open-label trial as blinding of treating team and patients was not possible. The setting during endotracheal intubation was different in two groups, ER (in most cases) in orotracheal group and PICU in nasotracheal group, which is more of a controlled environment. In our units (ER and PICU), all cases underwent endotracheal intubation through orotracheal route first as per the routine practice. Children randomized to nasotracheal route were extubated and then re-intubation through nasal route. Hence, we could not enroll cases before endotracheal intubation and then randomizing them directly to orotracheal or nasotracheal groups. In nasotracheal group, the act of extubation and reintubation through nasal route at the time of enrolment can be a confounder as the number of airway manipulations may had a bearing on the occurrence of PEAO. The long-term outcome after discharge from the PICU was not available, as it was not the part of this study. We could enroll only $38.9 \%$ (70 out of 180 ) of the calculated sample size. To have an adequate answer to the study question, large randomized trial with adequate sample size is needed to assess the impact of nasotracheal intubation on PEAO, other

\section{REFERENCES}

1. Folino TB, McKean G, Parks LJ. Nasotracheal Intubation. Treasure Island, FL: StatPearls (2021)

2. Holzapfel L. Nasal vs. oral intubation. Minerva Anestesiol. (2003) 69:348-52.

3. McMillan DD, Rademaker AW, Buchan KA, Reid A, Machin G, Sauve RS. Benefits of orotracheal and nasotracheal intubation in neonates requiring ventilatory assistance. Pediatrics. (1986) 77:39-44. doi: 10.1016/0883-9441(87)90137-7

4. Deutschman CS, Wilton P, Sinow J, Dibbell D. Jr., Konstantinides FN, Cerra FB. Paranasal sinusitis associated with nasotracheal intubation: a frequently unrecognized and treatable source of sepsis. Crit Care Med. (1986) 14:111-4.

5. Holzapfel L, Chevret S, Madinier G, Ohen F, Demingeon G, Coupry $\mathrm{A}$, et al. Influence of long-term oro- or nasotracheal intubation on nosocomial maxillary sinusitis and pneumonia: results of a prospective, randomized, clinical trial. Crit Care Med. (1993) 21:1132-8. doi: 10.1097/00003246-199308000-00010 clinical outcomes, and safety among children receiving long-term mechanical ventilation.

\section{CONCLUSION}

In this open-label RCT involving critically ill children undergoing mechanical ventilation, we noted that the rate of PEAO was similar in nasotracheal and orotracheal intubation groups. Slower recruitment rate and enrolment of lesser than required sample size are the major limitations.

\section{DATA AVAILABILITY STATEMENT}

The raw data supporting the conclusions of this article will be made available by the authors, on reasonable request.

\section{ETHICS STATEMENT}

The studies involving human participants were reviewed and approved by Institute Ethics Committee, PGIMER, Chandigarh, India. Written informed consent to participate in this study was provided by the participants' legal guardian/next of kin.

\section{AUTHOR CONTRIBUTIONS}

VK prepared the protocol, enrolled cases, collected data, reviewed the literature, and prepared the initial draft of the manuscript. SA conceptualized the study, supervised the preparation of the protocol and conduct of the study, analyzed data, and critically reviewed and finalized the manuscript. AKB supervised the data collection and helped in statistical analysis. KN supervised the data collection and performed literature review. All authors approved the final manuscript.

\section{SUPPLEMENTARY MATERIAL}

The Supplementary Material for this article can be found online at: https://www.frontiersin.org/articles/10.3389/fped. 2021.713516/full\#supplementary-material

6. van Zanten AR, Dixon JM, Nipshagen MD, de Bree R, Girbes AR, Polderman $\mathrm{KH}$. Hospital-acquired sinusitis is a common cause of fever of unknown origin in orotracheally intubated critically ill patients. Crit Care. (2005) 9:R583-90. doi: 10.1186/cc3805

7. Michelson A, Kamp HD, Schuster B. Sinusitis in long-term intubated, intensive care patients: nasal versus oral intubation. Anaesthesist. (1991) 40:100-4.

8. Michelson A, Schuster B, Kamp HD. Paranasal sinusitis associated with nasotracheal and orotracheal long-term intubation. Arch Otolaryngol Head Neck Surg. (1992) 118:937-9. doi: 10.1001/archotol.1992.018800900 53016

9. Bach A, Boehrer H, Schmidt H, Geiss HK. Nosocomial sinusitis in ventilated patients. Nasotracheal versus orotracheal intubation. Anaesthesia. (1992) 47:335-9. doi: 10.1111/j.1365-2044.1992.tb02177.x

10. Moore BM, Blumberg K, Laguna TA, Liu M, Zielinski EE, Kurachek SC. Incidental sinusitis in a pediatric intensive care unit. Pediatr Crit Care Med. (2012) 13:e64-8. doi: 10.1097/PCC.0b013e31820ac3f5 
11. Christian CE, Thompson NE, Wakeham MK. Use and outcomes of nasotracheal intubation among patients requiring mechanical ventilation across U S PICUs. Pediatr Crit Care Med. (2020) 21:620-4. doi: 10.1097/PCC.0000000000002267

12. Esteban A, Anzueto A, Alia I, Gordo F, Apezteguia C, Palizas F, et al. How is mechanical ventilation employed in the intensive care unit? An international utilization review. Am J Respir Crit Care Med. (2000) 161:14508. doi: 10.1164/ajrccm.161.5.9902018

13. Hong SB, Oh BJ, Kim YS, Kang EH, Kim CH, Park YB, et al. Characteristics of mechanical ventilation employed in intensive care units: a multicenter survey of hospitals. J Korean Med Sci. (2008) 23:94853. doi: 10.3346/jkms.2008.23.6.948

14. Nishisaki A, Turner DA, Brown CA, III, Walls RM, Nadkarni VM, National Emergency Airway Registry for C, et al. A National Emergency Airway Registry for children: landscape of tracheal intubation in 15 PICUs. Crit Care Med. (2013) 41:874-85. doi: 10.1097/CCM.0b013e3182746736

15. Lee JH, Nuthall G, Ikeyama T, Saito O, Mok YH, Shepherd M, et al. Tracheal intubation practice and safety across international PICUs: a report from national emergency airway registry for children. Pediatr Crit Care Med. (2019) 20:1-8. doi: 10.1097/PCC.0000000000001782

16. Kambestad KK, Huack A, Nair S, Chapman R, Chin S, Langga L, et al. The adverse impact of unplanned extubation in a cohort of critically ill neonates. Respir Care. (2019) 64:1500-7. doi: 10.4187/respcare.06721

17. Kanthimathinathan HK, Durward A, Nyman A, Murdoch IA, Tibby SM. Unplanned extubation in a paediatric intensive care unit: prospective cohort study. Intensive Care Med. (2015) 41:1299-306. doi: 10.1007/s00134-015-3872-4

18. Roddy DJ, Spaeder MC, Pastor W, Stockwell DC, Klugman D. Unplanned extubations in children: impact on hospital cost and length of stay. Pediatr Crit Care Med. (2015) 16:572-5. doi: 10.1097/PCC.0000000000000406

19. Davis D, Barbee L, Ririe D. Pediatric endotracheal tube selection: a comparison of age-based and height-based criteria. AANA J. (1998) 66:299-303.

20. Baranwal AK, Meena JP, Singhi SC, Muralidharan J. Dexamethasone pretreatment for $24 \mathrm{~h}$ versus $6 \mathrm{~h}$ for prevention of postextubation airway obstruction in children: a randomized double-blind trial. Intensive Care Med. (2014) 40:1285-94. doi: 10.1007/s00134-014-3358-9

21. Parajuli B, Baranwal AK, Kumar MP, Jayashree M, Takia L. Twenty-fourhour pretreatment with low dose $(0.25 \mathrm{mg} / \mathrm{kg} /$ dose $)$ versus high dose $(0.5$ $\mathrm{mg} / \mathrm{kg} /$ dose) dexamethasone in reducing the risk of postextubation airway obstruction in children: a randomized open-label noninferiority trial. Pediatr Pulmonol. (2021) 56:2292-301. doi: 10.22541/au.159170794.47022777

22. Westley CR, Cotton EK, Brooks JG. Nebulized racemic epinephrine by IPPB for the treatment of croup: a double-blind study. Am J Dis Child. (1978) 132:484-7. doi: 10.1001/archpedi.1978.02120300044008

23. Samprathi M, Baranwal AK, Gupta PK, Jayashree M. Pre-extubation ultrasonographic measurement of intracricoid peritubal free space: a pilot study to predict post-extubation airway obstruction in children. Int J Pediatr Otorhinolaryngol. (2020) 138:110348. doi: 10.1016/j.ijporl.2020.110348

24. Goldstein B, Giroir B, Randolph A. International Consensus Conference on Pediatric S. International pediatric sepsis consensus conference: definitions for sepsis and organ dysfunction in paediatrics. Pediatr Crit Care Med. (2005) 6:2-8. doi: 10.1097/01.PCC.0000149131.72248.E6

25. Koenig SM, Truwit JD. Ventilator-associated pneumonia: diagnosis, treatment, and prevention. Clin Microbiol Rev. (2006) 19:637-57. doi: 10.1128/CMR.00051-05

26. Edsberg LE, Black JM, Goldberg M, McNichol L, Moore L, Sieggreen M. Revised national pressure ulcer advisory panel pressure injury staging system: revised pressure injury staging system. J Wound Ostomy Continence Nurs. (2016) 43:585-97. doi: 10.1097/WON.0000000000000281

27. Veder LL, Joosten KFM, Schlink K, Timmerman MK, Hoeve LJ, van der Schroeff MP, et al. Post-extubation stridor after prolonged intubation in the pediatric intensive care unit (PICU): a prospective observational cohort study. Eur Arch Otorhinolaryngol. (2020) 277:1725-31. doi: 10.1007/s00405-020-05877-0

28. Sinha A, Jayashree M, Singhi S. Aerosolized L-epinephrine vs. budesonide for post extubation stridor: a randomized controlled trial. Indian Pediatr. (2010) 47:317-22. doi: 10.1007/s13312-010 0060-z

29. Kimura S, Ahn JB, Takahashi M, Kwon S, Papatheodorou S. Effectiveness of corticosteroids for post-extubation stridor and extubation failure in pediatric patients: a systematic review and meta-analysis. Ann Intensive Care. (2020) 10:155. doi: 10.1186/s13613-020-00773-6

30. DeMichele JC, Vajaria N, Wang H, Sweeney DM, Powers KS, Cholette JM. Cuffed endotracheal tubes in neonates and infants undergoing cardiac surgery are not associated with airway complications. J Clin Anesth. (2016) 33:4227. doi: 10.1016/j.jclinane.2016.04.038

31. Chand R, Roy Chowdhury S, Rupert E, Mandal CK, Narayan P. Benefits of using high-volume-low-pressure tracheal tube in children undergoing congenital cardiac surgery: evidence from a prospective randomized study. Semin Cardiothorac Vasc Anesth. (2018) 22:3005. doi: 10.1177/1089253217750753

32. Depoix JP, Malbezin S, Videcoq M, Hazebroucq J, Barbier-Bohm G, Gauzit R, et al. Oral intubation v. nasal intubation in adult cardiac surgery. Br J Anaesth. (1987) 59:167-9. doi: 10.1093/bja/59.2.167

33. Chahar JS, Das PK, Dubey RK, Malviya D, Harjai M, Rastogi S. Comparison of orotracheal versus nasotracheal fiberoptic intubation using hemodynamic parameters in patients with anticipated difficult airway. Anesth Essays Res. (2020) 14:81-6. doi: 10.4103/aer.AER_6_20

34. Bowman JP, Nedley MP, Jenkins KA, Fahncke CR. Pilot study comparing nasal vs. oral intubation for dental surgery by physicians, nurse anesthetists, and trainees. Anesth Prog. (2018) 65:89-93. doi: 10.2344/anpr-65-02-07

35. Spence K, Barr P. Nasal versus oral intubation for mechanical ventilation of newborn infants. Cochrane Database Syst Rev. (2000) 2:CD000948. doi: 10.1002/14651858.CD000948

36. Bouza C, Garcia E, Diaz M, Segovia E, Rodriguez I. Unplanned extubation in orally intubated medical patients in the intensive care unit: a prospective cohort study. Heart Lung. (2007) 36:270-6. doi: 10.1016/j.hrtlng.2006. 10.002

37. Klugman D, Melton K, Maynord PO, Dawson A, Madhavan G, Montgomery VL, et al. Assessment of an unplanned extubation bundle to reduce unplanned extubations in critically ill neonates, infants, and children. JAMA Pediatr. (2020) 174:e200268. doi: 10.1001/jamapediatrics.2020.0268

38. Chevron V, Menard JF, Richard JC, Girault C, Leroy J, Bonmarchand G. Unplanned extubation: risk factors of development and predictive criteria for reintubation. Crit Care Med. (1998) 26:1049-53. doi: 10.1097/00003246-199806000-00026

39. Listello D, Sessler CN. Unplanned extubation. Clin Predict Reintub Chest. (1994) 105:1496-503. doi: 10.1378/chest.105.5.1496

40. Piva JP, Amantea S, Luchese S, Giugno K, Maia TR, Einloft L. Accidental extubation in a pediatric intensive care unit. J Pediatr (Rio J). (1995) 71:726. doi: 10.2223/JPED.707

41. Aebert H, Hunefeld G, Regel G. Paranasal sinusitis and sepsis in ICU patients with nasotracheal intubation. Intensive Care Med. (1988) 15:2730. doi: 10.1007/BF00255632

Conflict of Interest: The authors declare that the research was conducted in the absence of any commercial or financial relationships that could be construed as a potential conflict of interest.

Publisher's Note: All claims expressed in this article are solely those of the authors and do not necessarily represent those of their affiliated organizations, or those of the publisher, the editors and the reviewers. Any product that may be evaluated in this article, or claim that may be made by its manufacturer, is not guaranteed or endorsed by the publisher.

Copyright (C) 2021 Kumar, Angurana, Baranwal and Nallasamy. This is an openaccess article distributed under the terms of the Creative Commons Attribution License (CC BY). The use, distribution or reproduction in other forums is permitted, provided the original author(s) and the copyright owner(s) are credited and that the original publication in this journal is cited, in accordance with accepted academic practice. No use, distribution or reproduction is permitted which does not comply with these terms. 